MATHEMATICS OF COMPUTATION

Volume 69, Number 229, Pages 339-349

S 0025-5718(99)01167-9

Article electronically published on September 8, 1999

\title{
ON FUNDAMENTAL DOMAINS OF ARITHMETIC FUCHSIAN GROUPS
}

\author{
STEFAN JOHANSSON
}

\begin{abstract}
Let $K$ be a totally real algebraic number field and $\mathcal{O}$ an order in a quaternion algebra $\mathfrak{A}$ over $K$. Assume that the group $\mathcal{O}^{1}$ of units in $\mathcal{O}$ with reduced norm equal to 1 is embedded into $P S L_{2}(\mathbb{R})$ as an arithmetic Fuchsian group. It is shown how Ford's algorithm can be effectively applied in order to determine a fundamental domain of $\mathcal{O}^{1}$ as well as a complete system of generators of $\mathcal{O}^{1}$.
\end{abstract}

\section{INTRODUCTION}

Let $K$ be a totally real algebraic number field of degree $n$ over $\mathbb{Q}$. Let $R$ be the ring of integers in $K$ and $\left\{\sigma_{1}, \ldots, \sigma_{n}\right\}$ the $n$ different embeddings of $K$ into $\mathbb{R}$. Assume that $\mathfrak{A}$ is a quaternion algebra over $K$ such that

$$
\mathfrak{A} \otimes \mathbb{Q} \mathbb{R} \cong M_{2}(\mathbb{R}) \times \mathbb{H}^{n-1},
$$

where $\mathbb{H}$ is the Hamiltonian quaternion algebra. We fix $\sigma_{1}$ to be the only embedding of $K$ into $\mathbb{R}$ such that

$$
\mathfrak{A} \otimes_{K_{\sigma_{1}}} \mathbb{R} \cong M_{2}(\mathbb{R}) .
$$

We will throughout this paper assume that $\mathfrak{A}$ is a division algebra, that is, $\mathfrak{A} \neq$ $M_{2}(K)$.

An order $\mathcal{O}$ in $\mathfrak{A}$ is a subring of $\mathfrak{A}$ containing $R$, which is finitely generated as an $R$-module and such that $K \mathcal{O}=\mathfrak{A}$. We consider the group

$$
\overline{\mathcal{O}}^{1}=\{x \in \mathcal{O}: N(x)=1\} /\{ \pm 1\},
$$

where $N$ is the reduced norm from $\mathfrak{A}$ to $K$. The map $\sigma_{1}$ induces an embedding of $\overline{\mathcal{O}}^{1}$ as a discrete subgroup in $P S L_{2}(\mathbb{R})=S L_{2}(\mathbb{R}) /\{ \pm 1\}$. Such a discrete subgroup (or any commensurable with such) is called an arithmetic Fuchsian group.

The group $S L_{2}(\mathbb{R})$ acts on the complex upper half-plane $\mathcal{H}$ by Möbius transformations

$$
g \cdot z=\frac{a z+b}{c z+d}, \text { where } g=\left[\begin{array}{ll}
a & b \\
c & d
\end{array}\right] \in S L_{2}(\mathbb{R}) .
$$

Since the action of -1 is trivial, we get an action by $P S L_{2}(\mathbb{R})$. If $\Gamma$ is a discrete subgroup of $P S L_{2}(\mathbb{R})$, then it is possible to provide the quotient space $\Gamma \backslash \mathcal{H}$ with the structure of a Riemann surface.

Received by the editor June 4, 1997.

1991 Mathematics Subject Classification. Primary 11F06, 20H10; Secondary 11R52.

Key words and phrases. Arithmetic Fuchsian groups, quaternion orders, fundamental domains.

(C)1999 American Mathematical Society 
The main aim of this paper is to describe an algorithm, which finds a fundamental domain of $\Gamma$ in $\mathcal{H}$, when $\Gamma=\overline{\mathcal{O}}^{1}$ for an order $\mathcal{O}$ in a quaternion division algebra. In this case $\Gamma \backslash \mathcal{H}$ is compact, since $\overline{\mathcal{O}}^{1}$ does not contain any parabolic elements. The basis of the algorithm is Ford's classical method [3]. However, the observation that this can be used in the present case to give an efficient algorithm seems to be little known, at least when $K \neq \mathbb{Q}$.

In Section 1, we recall the basic facts about quaternion algebras that are needed in the paper. Section 2 contains the general idea of our algorithm. In Section 3, we derive an explicit matrix representation of a maximal order in an arbitrary indefinite quaternion algebra over $\mathbb{Q}$ with given discriminant. Such representations already exist in the literature, but ours is very simple and well-suited for calculations. By using this representation, we get a completely explicit algorithm that finds a fundamental domain when $\mathcal{O}$ is a maximal order in an arbitrary indefinite quaternion algebra over $\mathbb{Q}$. For a non-maximal order $\mathcal{O}$, it suffices to find a basis of $\mathcal{O}$ in a maximal order. The case $K=\mathbb{Q}$ is different and much easier than the general case. Therefore, we illustrate the general case in Section 4 by giving a careful explanation when $K$ is a quadratic field. We conclude the paper with some remarks and examples.

\section{Quaternion algebras}

It is well-known that one can always find a basis $1, j, k, j k$ for $\mathfrak{A}$ over $K$ which satisfies

$$
j^{2}=a, k^{2}=b \text { and } j k=-k j, \quad \text { where } a, b \in K, a b \neq 0 .
$$

We will denote this algebra by $(a, b)_{K}$. It can be embedded in $M_{2}(K(\sqrt{a})$ ) (or $\left.M_{2}(K(\sqrt{b}))\right)$ for example by

$$
i \longmapsto\left[\begin{array}{cc}
\sqrt{a} & 0 \\
0 & -\sqrt{a}
\end{array}\right] \quad \text { and } \quad j \longmapsto\left[\begin{array}{ll}
0 & b \\
1 & 0
\end{array}\right] .
$$

There is a natural involution in $(a, b)_{K}$, which in this basis is given by

$$
x=x_{0}+x_{1} j+x_{2} k+x_{3} j k \longmapsto \bar{x}=x_{0}-x_{1} j-x_{2} k-x_{3} j k .
$$

The (reduced) trace and (reduced) norm are defined by

$$
\operatorname{Tr}(x)=x+\bar{x} \quad \text { and } \quad N(x)=x \bar{x} .
$$

If we evaluate the norm, we see that it is the quadratic form

$$
N(x)=x_{0}^{2}-a x_{1}^{2}-b x_{2}^{2}+a b x_{3}^{2} .
$$

In order to get control over the different isomorphism classes of quaternion algebras over $K$, one considers the completions $\mathfrak{A}_{\nu}=\mathfrak{A} \otimes_{K} K_{\nu}$. Here $K_{\nu}$ is a completion of $K$ corresponding either to a prime ideal, or to one of the infinite (real) embeddings. It is well-known that for every $\mathfrak{A}_{\nu}$ there are only two possibilities: $\mathfrak{A}_{\nu} \cong M_{2}\left(K_{\nu}\right)(\mathfrak{A}$ splits at $\nu)$ or $\mathfrak{A}_{\nu} \cong \mathbb{H}_{\nu}(\mathfrak{A}$ is ramified at $\nu)$, where $\mathbb{H}_{\nu}$ is a division algebra over $K_{\nu}$ unique up to isomorphism. The following classical result of Hasse solves the problem of classifying quaternion algebras up to isomorphism $[7, \S \S 57,71,72]$.

Theorem 1.1. Two quaternion algebras over $K$ are isomorphic iff they are ramified at the same places. Every algebra is ramified at an even number of places. 
Conversely given an even number of places, it is possible to find a quaternion algebra which is ramified exactly at those places.

It is obvious that $(a, b)_{\mathbb{R}}$ is ramified iff both $a$ and $b$ are negative. In order to decide whether $(a, b)_{K_{\nu}}$ is ramified when $\nu=\mathfrak{p}$ is a prime ideal, it is convenient to use the Hilbert symbol $\left(\frac{a, b}{\mathfrak{p}}\right)$ (see $\left.[7, \S 63]\right)$. The algebra $(a, b)_{K}$ is ramified at $\mathfrak{p}$ iff $\left(\frac{a, b}{\mathfrak{p}}\right)=-1$.

We define the discriminant $d(\mathfrak{A})$ of $\mathfrak{A}$ to be the product of the prime ideals at which $\mathfrak{A}$ is ramified. Let $\mathcal{O}$ be an order in $\mathfrak{A}$. Then the discriminant $d(\mathcal{O})$ of $\mathcal{O}$ is defined to be the square root of the $R$-ideal generated by all $\operatorname{det}\left(\operatorname{Tr}\left(x_{i} \bar{x}_{j}\right)\right)$, where $\left\{x_{1}, x_{2}, x_{3}, x_{4}\right\} \subset \mathcal{O}$. If $\mathcal{M}$ is a maximal order in $\mathfrak{A}$ containing $\mathcal{O}$, then the discriminants satisfy

$$
d(\mathcal{O})=d(\mathcal{M}) \cdot[\mathcal{M}: \mathcal{O}] \text { and } d(\mathcal{M})=d(\mathfrak{A}) .
$$

If $\mathfrak{A} \cong(a, b)_{K}$, then $\mathfrak{A}_{\sigma_{i}} \cong\left(\sigma_{i}(a), \sigma_{i}(b)\right)_{\mathbb{R}}$ for all $1 \leq i \leq n$. We identify $K$ with its image $\sigma_{1}(K)$ in $\mathbb{R}$. Hence our restriction (0.1) on the splitting of $\mathfrak{A}$ is equivalent to at least one of the two numbers $\{a, b\}$ being positive, but both numbers being negative for all pairs $\left\{\sigma_{i}(a), \sigma_{i}(b)\right\}$ with $i>1$.

\section{General case}

First we fix an embedding of $\overline{\mathcal{O}}^{1}$ in $P S L_{2}(\mathbb{R})$. We denote the image of $\overline{\mathcal{O}}^{1}$ in $P S L_{2}(\mathbb{R})$ by $\Gamma^{\prime}$. We choose this in such a way that $i \in \mathcal{H}$ is not a fixed point of any nontrivial element in $\Gamma^{\prime}$. The Möbius transformation given by the matrix

$$
\varphi=\left[\begin{array}{ll}
i & 1 \\
1 & i
\end{array}\right]
$$

maps $\mathcal{H}$ to the unit disk $\mathcal{U}$, and in particular $i$ to the origin. The action of $S L_{2}(\mathbb{R})$ on $\mathcal{H}$ transforms to an action of $S U(1,1)$ on $\mathcal{U}$, since

$$
S U(1,1)=\varphi \cdot S L_{2}(\mathbb{R}) \cdot \varphi^{-1} .
$$

The conjugation of $\Gamma^{\prime}$ by $\varphi$ gives an embedding $\Gamma$ of $\overline{\mathcal{O}}^{1}$ in $S U(1,1)$. Since the origin is not a fixed point of any nontrivial element in $\Gamma$, it is possible to use Ford's method to find a fundamental domain in $\mathcal{U}$. This method relies on Theorem 2.1 below.

First, recall that if

$$
g=\left[\begin{array}{ll}
a & b \\
c & d
\end{array}\right] \in S U(1,1) \quad \text { or } \quad g \in S L_{2}(\mathbb{R})
$$

with $c \neq 0$, then the isometric circle $C_{g}$ of $g$ is defined by

$$
C_{g}=\{z \in \mathbb{C}:|c z+d|=1\} .
$$

The geometric meaning of this is that $C_{g}$ is the set of points where $g$ acts as an Euclidean isometry. The following theorem was first proved by Ford in [3].

Theorem 2.1. Let $\Gamma$ be a discrete subgroup of $S U(1,1)$ such that the origin is not a fixed point of any nontrivial element in $\Gamma$. Let $C_{g}$ be the isometric circle of $g$. If $C_{g}^{o}$ is the set of all points outside $C_{g}$, then

$$
F=\mathcal{U} \cap \bigcap_{g \in \Gamma} C_{g}^{o}
$$

is a fundamental domain of $\Gamma$. 
We remark that $\varphi^{-1} F$ is obviously a fundamental domain in $\mathcal{H}$.

It is trivial to check that the origin of every isometric circle is outside of $\mathcal{U}$. Let $\Gamma_{\epsilon}$ be the set of all elements $g \in \Gamma$ for which the radius of $C_{g}$ is greater than $\epsilon$. The set of different isometric circles $C_{g}$ with $g \in \Gamma_{\epsilon}$ is finite for every discrete group $\Gamma$ and every positive number $\epsilon$. Now if

$$
F_{\epsilon}=\mathcal{U} \cap \bigcap_{g \in \Gamma_{\epsilon}} C_{g}^{o}, \quad \mathcal{U}_{\epsilon}=\{z \in \mathbb{C}:|z|<1-\epsilon\}, \quad F_{\epsilon} \subseteq \mathcal{U}_{\epsilon},
$$

then $F_{\epsilon}$ is a fundamental domain of $\Gamma$, since the origin of every isometric circle is outside of $\mathcal{U}$. This will eventually be the case for some $\epsilon>0$, since there are no parabolic elements and the hyperbolic area of a fundamental domain is finite in our case. Our goal is now to determine $\Gamma_{\epsilon}$.

Let $g$ be an element in $\Gamma^{\prime} \subset P S L_{2}(\mathbb{R})$, and suppose that

$$
g=\left[\begin{array}{ll}
a & b \\
c & d
\end{array}\right] .
$$

If $r_{g}$ denotes the radius of the isometric circle of $\varphi g \varphi^{-1} \in \Gamma$, then a direct calculation gives

$$
r_{g}=\frac{2}{\sqrt{(a-d)^{2}+(b+c)^{2}}}=\frac{2}{\sqrt{a^{2}+b^{2}+c^{2}+d^{2}-2}} .
$$

Hence a restriction $r_{g}>\epsilon$ gives an upper bound on the absolute value of the entries of $g$.

Since the norm is positive definite in all the other $n-1$ embeddings, we get upper bounds on the absolute values of $\sigma_{i}(a), \sigma_{i}(b), \sigma_{i}(c), \sigma_{i}(d)$ for $2 \leq i \leq n$. Now since we have bounds on all the $n$ different real embeddings of the entries in $\Gamma$, we get that we only have to try a finite number of elements for the entries of $g$ in order to find $\Gamma_{\epsilon}$.

When we have found $\Gamma_{\epsilon}$, we have in general an unnecessarily large set. Therefore it is convenient to determine the subset

$$
\Gamma_{\epsilon}^{\prime}=\left\{g \in \Gamma_{\epsilon}: C_{g} \not \subset \bigcup_{h \in \Gamma_{\epsilon}, h \neq g} C_{h}\right\} .
$$

Then we clearly have

$$
F_{\epsilon}=F_{\epsilon}^{\prime}:=\mathcal{U} \cap \bigcap_{g \in \Gamma_{\epsilon}^{\prime}} C_{g}^{o} .
$$

If $F_{\epsilon} \subseteq \mathcal{U}_{\epsilon}$, then we have a fundamental domain. Otherwise, we have to take a smaller $\epsilon$ and continue our search.

The realization of these observations is probably best explained by a detailed examination of the special case $n=2$. But first we will give a totally explicit solution in the case when $K=\mathbb{Q}$ and $\mathcal{O}$ is a maximal order.

\section{Rational CASE}

We begin with a solution to the representation problem of an indefinite quaternion algebra with given discriminant.

Proposition 3.1. Let $\mathfrak{A}$ be an indefinite quaternion algebra over $\mathbb{Q}$, with discriminant $d=p_{1} \cdots p_{2 r}$. Choose $p$ to be a prime such that $p \equiv 5(\bmod 8)$ and $\left(\frac{p}{p_{i}}\right)=-1$, $\forall p_{i}>2$. Then $\mathfrak{A} \cong(p, d)_{\mathbb{Q}}$. 
Proof. The Chinese Remainder Theorem and the Dirichlet Theorem about primes in linear progressions assure the existence of infinitely many prime numbers $p$ fulfilling the requirements. Calculating the Hilbert symbol gives

$$
\left(\frac{p, d}{p_{i}}\right)=\left(\frac{p, p_{i}}{p_{i}}\right)=\left(\frac{p}{p_{i}}\right)=-1, \text { if } p_{i}>2 .
$$

Since $\left(\frac{2}{p}\right)=-1$, while $p \equiv 5(\bmod 8)$ and $\left(\frac{p}{p_{i}}\right)\left(\frac{p_{i}}{p}\right)=1$, while $p \equiv 1(\bmod 4)$, we also have

$$
\left(\frac{p, d}{p}\right)=\prod_{i=1}^{2 r}\left(\frac{p_{i}}{p}\right)=1
$$

Hence $(p, d)_{\mathbb{Q}}$ is ramified at all odd prime numbers dividing $d$ and not at $p$. Since it is not ramified at any other odd prime and ramified at an even number of places, it has to have the same discriminant as $\mathfrak{A}$. Hence $\mathfrak{A}$ is isomorphic to $(p, d)_{\mathbb{Q}}$.

Now suppose that $\mathfrak{A}$ has discriminant $d$ and that $p$ is a prime number satisfying the conditions in Proposition 3.1. Let $\mathcal{O}_{1}$ be the order with $\mathbb{Z}$-basis $1, j, k, j k$, where

$$
j^{2}=p, \quad k^{2}=d, \quad j k=-k j .
$$

Then the discriminant of $\mathcal{O}_{1}$ is equal to $4 p d$. Let $m$ be an integer such that $d \equiv$ $m^{2}(\bmod p)$. It exists since $\left(\frac{d}{p}\right)=1$. The two elements

$$
e_{1}=\frac{1+j}{2} \quad \text { and } \quad e_{2}=\frac{m j+j k}{p},
$$

as well as their products, all have integer norms and traces. Hence

$$
\mathcal{O}=\mathbb{Z}+\mathbb{Z} e_{1}+\mathbb{Z} e_{2}+\mathbb{Z} e_{1} e_{2}
$$

is an order. Furthermore, since a direct computation gives $\left[\mathcal{O}: \mathcal{O}_{1}\right]=4 p$, we get that $\mathcal{O}$ is a maximal order in $\mathfrak{A}$. Since all maximal orders in an indefinite quaternion algebra over $\mathbb{Q}$ are conjugate [2], we have proved:

Proposition 3.2. Let $\mathfrak{A}$ be as in Proposition 3.1. Then every maximal order in $\mathfrak{A}$ is conjugate to $\mathcal{O}=\mathbb{Z}+\mathbb{Z} e_{1}+\mathbb{Z} e_{2}+\mathbb{Z} e_{1} e_{2}$, where $e_{1}$ and $e_{2}$ are defined by (3.1).

There are several more complicated presentations analogous to Proposition 3.2 in the literature. See for example [6].

We now fix an embedding in $M_{2}(\mathbb{R})$ by setting

$$
j=\left[\begin{array}{cc}
\sqrt{p} & 0 \\
0 & -\sqrt{p}
\end{array}\right] \text { and } k=\left[\begin{array}{cc}
0 & \sqrt{d} \\
\sqrt{d} & 0
\end{array}\right] .
$$

Then it is straightforward to show that $\mathcal{O}$ consists of all elements $g$ such that

where

$$
g=\frac{1}{2 p}\left[\begin{array}{cc}
p x+y \sqrt{p} & \sqrt{d}(p z+v \sqrt{p}) \\
\sqrt{d}(p z-v \sqrt{p}) & p x-y \sqrt{p}
\end{array}\right],
$$

We get that

$$
N(g)=\frac{1}{4 p}\left(p x^{2}-y^{2}-d p z^{2}+d v^{2}\right),
$$

and observe that $i$ is not a fixed point for any $g \in \overline{\mathcal{O}}^{1}$, since $p x^{2}+d v^{2}=4 p$ has no integer solutions except $x= \pm 2, v=0$. If we let $s=y^{2}+d p z^{2}$, then $N(g)=1$ 
implies $p x^{2}+d v^{2}=4 p+s$ and $s>0$ if $g \neq \pm 1$. The radius $r_{g}$ of the isometric circle of $\varphi(g)$ is closely related to the norm, because we get

$$
r_{g}=\frac{2}{\sqrt{\frac{1}{2 p}\left(p x^{2}+y^{2}+d p z^{2}+d v^{2}\right)-2}}=\frac{2 \sqrt{p}}{\sqrt{s}} .
$$

Hence by searching for integer solutions satisfying (3.2) to the equations

$$
s=y^{2}+d p z^{2} \quad \text { and } \quad 4 p+s=p x^{2}+d v^{2}
$$

for increasing parameter $s$, we get a listing of the elements in $\overline{\mathcal{O}}^{1}$ with decreasing radius $r_{g}$. This idea can be found in $[5,(5.6)]$ for a special case.

\section{Quadratic case}

We now turn to the case when $K=\mathbb{Q}(\sqrt{m})$, where $m$ is a positive, square free integer. Let $\mathfrak{A}$ be a quaternion algebra over $K$ satisfying (0.1), and fix a basis as in $(1.1)$, such that $\mathfrak{A} \cong(t, s)_{K}$. We may suppose that $t>0$ and that $\sqrt{t} \notin K$. We fix an embedding of $\mathfrak{A}$ into $M_{2}(\mathbb{R})$ by letting

$$
j=\left[\begin{array}{cc}
\sqrt{t} & 0 \\
0 & -\sqrt{t}
\end{array}\right] \quad \text { and } \quad k=\left[\begin{array}{cc}
0 & r_{1} \\
r_{2} & 0
\end{array}\right],
$$

where $r_{1}, r_{2} \in \mathbb{R}$ are such that $s=r_{1} r_{2}$. If we choose $r_{1} \neq \pm r_{2}$, then $i$ is never a fixed point of any nontrivial element in an order $\mathcal{O}$ in $\mathfrak{A}$. However, if it is possible to choose $r_{1}= \pm r_{2}= \pm \sqrt{|s|}$ without $i$ being a fixed point, then this will give the most efficient bounds. Let $a=a_{1}+a_{2} \sqrt{m} \in \mathbb{Z}[\sqrt{m}$, and similarly for $b, c, d$. Now we see that any order in $\mathfrak{A}$ may be realized as all elements of the form

$$
g=\frac{1}{r}\left[\begin{array}{cc}
a+b \sqrt{t} & r_{1}(c+d \sqrt{t}) \\
r_{2}(c-d \sqrt{t}) & a-b \sqrt{t}
\end{array}\right],
$$

where $a_{i}, b_{i}, c_{i}, d_{i}$ satisfy some specific congruence conditions and $r \in \mathbb{Z}$. This of course is true in the general case if $a=\sum a_{i} \gamma_{i}$ and similarly for $b, c, d$, with $\left\{\gamma_{i}\right\}$ a basis of $K$ over $\mathbb{Q}$. The norm for $g$ is given by

$$
N(g)=\frac{1}{r^{2}}\left(a^{2}-t b^{2}-s c^{2}+s t d^{2}\right) .
$$

Now for the other embedding $\sigma_{2}$, for convenience we denote $\sigma_{2}(x)$ by $\tilde{x}$. If $N(g)=1$, then $N(\tilde{g})=1$ and hence

$$
r^{2}=\tilde{a}^{2}-\tilde{t} \tilde{b}^{2}-\tilde{s} \tilde{c}^{2}+\tilde{s} \tilde{t} \tilde{d}^{2} .
$$

Since both $\tilde{s}$ and $\tilde{t}$ are negative, this gives the following successive bounds:

$$
\left\{\begin{array}{l}
|\tilde{a}| \leq r \\
|\tilde{b}| \leq \sqrt{\frac{r^{2}-\tilde{a}^{2}}{-\tilde{t}}}, \\
|\tilde{d}| \leq \sqrt{\frac{r^{2}-\tilde{a}^{2}+\tilde{t}^{2}}{\tilde{\tilde{s} \tilde{b}}}}, \\
|\tilde{c}| \leq \sqrt{\frac{r^{2}-\tilde{a}^{2}+\tilde{t} \tilde{b}^{2}-\tilde{s} \tilde{t} \tilde{d}^{2}}{-\tilde{s}}}
\end{array}\right.
$$

In the general case $[K: \mathbb{Q}]=n$, we get $n-1$ systems of bounds like (4.2).

From (2.2), we get that $r_{g}=\frac{2}{\sqrt{q-2}}$, where

$$
q=\frac{1}{r^{2}}\left(2 a^{2}+2 t b^{2}+\frac{4 s^{2} t}{r_{1}^{2}+r_{2}^{2}} d^{2}+\left(r_{1}^{2}+r_{2}^{2}\right)\left(c+d \frac{r_{1}^{2}-r_{2}^{2}}{r_{1}^{2}+r_{2}^{2}} \sqrt{t}\right)^{2}\right) .
$$


Now $r_{g}>\epsilon$ is equivalent to $q<N_{\epsilon}:=2+\frac{4}{\epsilon^{2}}$, and $q<N_{\epsilon}$ implies the following bounds:

$$
\left\{\begin{array}{l}
|a|<r \sqrt{\frac{N_{\epsilon}}{2}} \\
|b|<\sqrt{\frac{1}{2 t}\left(r^{2} N_{\epsilon}-2 a^{2}\right)} \\
|d|<\sqrt{\frac{r_{1}^{2}+r_{2}^{2}}{4 s^{2} t}\left(r^{2} N_{\epsilon}-2 a^{2}-2 t b^{2}\right)} \\
|c|<\sqrt{\frac{1}{r_{1}^{2}+r_{2}^{2}}\left(r^{2} N_{\epsilon}-2 a^{2}-2 t b^{2}-\frac{4 s^{2} t}{r_{1}^{2}+r_{2}^{2}} d^{2}\right)}+\frac{\left|r_{1}^{2}-r_{2}^{2}\right|}{r_{1}^{2}+r_{2}^{2}} \sqrt{t}|d| .
\end{array}\right.
$$

The mean value of the bound on $|\tilde{a}|$ in (4.2) and the one on $|a|$ in (4.3) are bounds on the absolute value of $a_{1}$, and the corresponding holds for $b_{1}, c_{1}$ and $d_{1}$. Moreover the bounds in (4.2) give the following relative bounds:

$$
\frac{a_{1}-B_{a}}{\sqrt{d}} \leq a_{2} \leq \frac{a_{1}+B_{a}}{\sqrt{d}},
$$

where $B_{a}(=r)$ is the bound on $|\tilde{a}|$. Similar bounds hold for $b_{2}, c_{2}$ and $d_{2}$. These bounds give a finite set of integer vectors $\left(a_{1}, b_{1}, c_{1}, d_{1}, a_{2}, b_{2}, c_{2}, d_{2}\right)$ to check in order to get $\overline{\mathcal{O}}_{\epsilon}^{1}$. Since -1 is equal to 1 in $P S L_{2}(\mathbb{R})$, we may restrict to $a_{1} \geq 0$. In the general case, it might of course be more elaborate to determine bounds on $a_{i}, b_{i}, c_{i}$ and $d_{i}$, but there is no difficulty in doing it.

\section{Remarks And EXAMPles}

Before we conclude with some examples, we make a few remarks. The first one concerns a generalization to a wider family of arithmetic Fuchsian groups, and the second one gives a coarse estimate of the number $\epsilon$ in (2.1).

Remark 5.1. Let $U$ be the units of $R$, let $U^{+}$be the set of totally positive elements in $U$ and let $U^{2}$ be the subgroup of all squares in $U$. We have

$$
\overline{\mathcal{O}}^{1} \cong\left\{x \in \mathcal{O}: N(x) \in U^{2}\right\} / U,
$$

with an isomorphism given by $x \mapsto x / \sqrt{N(x)}$. Now let $E$ be any group such that $U^{2} \subseteq E \subseteq U^{+}$. Then the group

$$
\mathcal{O}_{E}=\{x \in \mathcal{O}: N(x) \in E\} / U
$$

acts on $\mathcal{H}$, and we get a Riemann surface $\mathcal{O}_{E} \backslash \mathcal{H}$. It is easy to adapt our method for this case. To do so, we choose representatives for the cosets of $U^{2}$ in $E$. Then we search for elements in $\mathcal{O}$ with norm among the representatives, instead of just for those with norm equal to one. We may have to adjust the bounds slightly, since the second equality in (2.2) is changed when the norm is not equal to one.

Remark 5.2. Let $\mathcal{O}$ be a maximal order in an algebra $\mathfrak{A}$ over $K$, and let $\mathcal{F}$ be a fundamental domain of $\mathcal{O}_{E}$ in $\mathcal{H}$. If $\mu$ denotes the normalized hyperbolic area on $\mathcal{H}$ corresponding to the form $\omega=\frac{d x d y}{2 \pi y^{2}}$, then we have [8]

$$
\mu(\mathcal{F})=(-1)^{n} \frac{\zeta_{K}(-1)}{2^{n-2}\left[E: U^{2}\right]} \prod_{\mathfrak{p} \mid d(\mathfrak{A})}(N \mathfrak{p}-1),
$$


where $N \mathfrak{p}$ is the norm of $\mathfrak{p}$, and $\zeta_{K}$ is the zeta function of $K$. If we are able to use this formula to calculate the area of a fundamental domain, then this will give us an idea on how to choose $\epsilon$. A direct calculation gives

$$
\mu\left(\mathcal{U}_{\epsilon}\right)=\frac{2(1-\epsilon)^{2}}{1-(1-\epsilon)^{2}},
$$

and $\mathcal{F} \subset \mathcal{U}_{\epsilon}$ implies

$$
\epsilon<\epsilon_{0}=1-\sqrt{\frac{\mu(\mathcal{F})}{4 \pi+\mu(\mathcal{F})}} .
$$

This is far from sufficient, and examples suggest that $\epsilon=\epsilon_{0} / 2$ is a sufficient choice when $\mu(\mathcal{F})$ is small $(<200)$. However, when $\mu(\mathcal{F})$ grows, then we have to choose $\epsilon=\epsilon_{0} / k$, where the factor $k$ is larger than 2 and grows with $\mu(\mathcal{F})$. It is the presence of big isometric circles that forces $\epsilon$ to be small.

Remark 5.3. A Ford fundamental domain also gives us a finite set of generators of $\mathcal{O}_{E}$-namely, the set of elements which correspond to the isometric circles which make up the sides of the fundamental domain. However, in general this set is not minimal.

We conclude with some explicit examples.

Example 5.4. First let $K=\mathbb{Q}(\sqrt{2})$ and let $p$ be a prime satisfying $p \equiv 3(\bmod 8)$. Thus $p$ remains prime in $K$. We adopt the notation from the beginning of Section 4 and set

$$
\left\{\begin{array}{l}
t=1+\sqrt{2}, s=-p, r=2, \\
a_{i} \equiv c_{i}(\bmod 2) \text { and } b_{i} \equiv d_{i}(\bmod 2) \text { for } i=1,2 .
\end{array}\right.
$$

Then it is easy to check that the discriminant of $\mathfrak{A}=(t, s)_{K}$ is equal to $p$, and that the elements satisfying (5.2) form a maximal order $\mathcal{O}$ in $\mathfrak{A}$. This is all the information we need to determine a fundamental domain for $\overline{\mathcal{O}}^{1}$ in $\mathcal{H}$. For example, in the special case $p=3$, we get the fundamental domain in Figure 1.

The point $D$ is the only elliptic point, and it is of order 3 . The generators and the sides which they identify are given in Table 1. From this we get that the genus of the surface is equal to 1 .

TABLE 1. Generators of the group corresponding to Figure 1.

\begin{tabular}{|c|c|c|c|c|c|c|c|c|}
\hline$a_{1}$ & $a_{2}$ & $b_{1}$ & $b_{2}$ & $c_{1}$ & $c_{2}$ & $d_{1}$ & $d_{2}$ & Identification \\
\hline 1 & 0 & 0 & 0 & 1 & 0 & 0 & 0 & $A D \longrightarrow A^{\prime} D$ \\
1 & 1 & 0 & -1 & -1 & -1 & 0 & -1 & $A B \longrightarrow A^{\prime \prime} B^{\prime \prime}$ \\
1 & 1 & 0 & 1 & 1 & 1 & 0 & 1 & $A^{\prime \prime} B^{\prime \prime} \longrightarrow A B$ \\
1 & 1 & 0 & 1 & -1 & -1 & 0 & -1 & $A^{\prime \prime} C^{\prime \prime} \longrightarrow A^{\prime} C^{\prime}$ \\
1 & 1 & 0 & -1 & 1 & 1 & 0 & 1 & $A^{\prime} C^{\prime} \longrightarrow A^{\prime \prime} C^{\prime \prime}$ \\
2 & 1 & -1 & 1 & -2 & -1 & -1 & -1 & $B^{\prime \prime} C \longrightarrow B^{\prime} C^{\prime}$ \\
2 & 1 & 1 & -1 & 2 & 1 & 1 & 1 & $B^{\prime} C^{\prime} \longrightarrow B^{\prime \prime} C$ \\
2 & 1 & 1 & -1 & -2 & -1 & -1 & -1 & $B C \longrightarrow B^{\prime} C^{\prime \prime}$ \\
2 & 1 & -1 & 1 & 2 & 1 & 1 & 1 & $B^{\prime} C^{\prime \prime} \longrightarrow B C$ \\
\hline
\end{tabular}




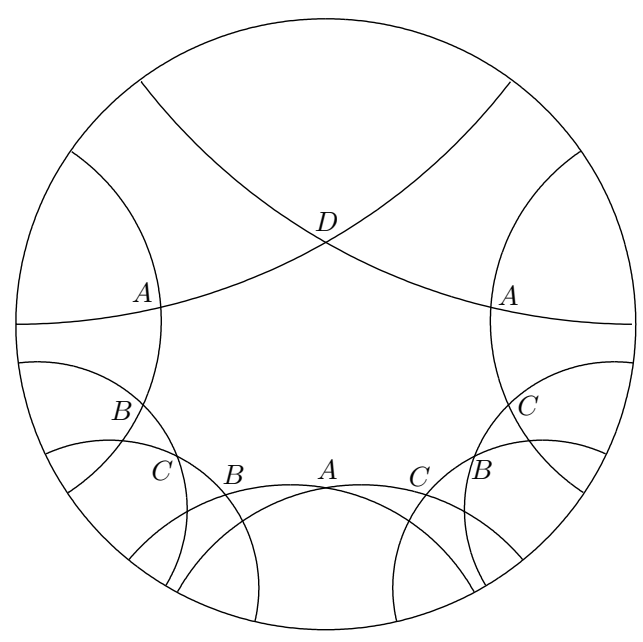

Figure 1. Fundamental domain in the case of a maximal order in $(1+\sqrt{2},-3)_{\mathbb{Q}(\sqrt{2})}$.

Example 5.5. The next example is well-known and was first constructed from a geometric point of view; see [1]. Again we take $K=\mathbb{Q}(\sqrt{2})$, but now let $t=\sqrt{2}-1, s=-1$ and $r=1$ and no congruence conditions. The order $\mathcal{O}$ in $\mathfrak{A}=(\sqrt{2}-1,-1)_{K}$ which we get has $d(\mathcal{O})=4$, and since $d(\mathfrak{A})=\sqrt{2}$ the index of $\mathcal{O}$ in a maximal order in $\mathfrak{A}$ is $2 \sqrt{2}$. Let $\Gamma$ be the subgroup of index 2 in $\overline{\mathcal{O}}^{1}$, which is defined by restricting to $a_{1}$ odd. Then the fundamental domain we get for $\Gamma$ is the regular octagon in Figure 2 with opposite sides identified.

Example 5.6. Now let $\omega$ be a root of an irreducible polynomial $f$ of degree 3 with integer coefficients, and let $K=\mathbb{Q}(\omega)$ be the cubic field generated by $\omega$. We suppose that $K$ is totally real, $R=\mathbb{Z}[\omega]$ is the ring of integers in $K$, and $\omega$ is a unit in $R$. Furthermore, assume that $\omega=\sigma_{1}(\omega)>0, \sigma_{2}(\omega)<0$ and $\sigma_{3}(\omega)<0$ for the 3

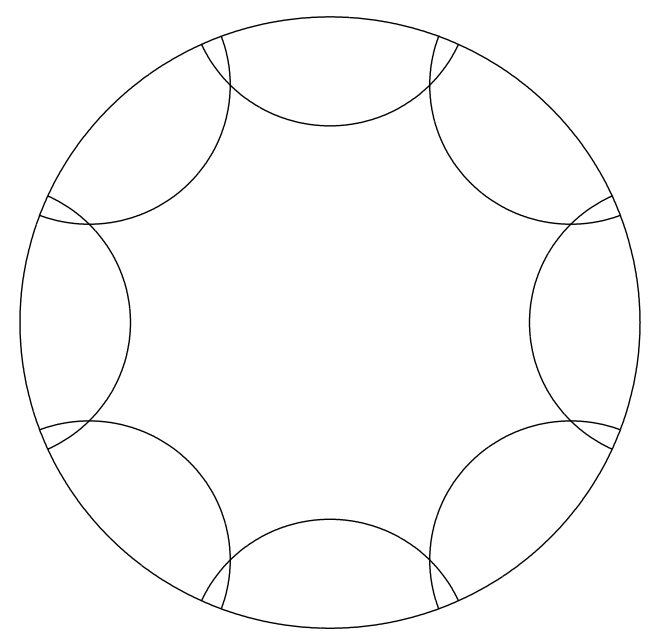

FIgURE 2. The regular octagon. 
different embeddings of $K$ in $\mathbb{R}$. We consider the quaternion algebra $\mathfrak{A}=(\omega,-1)_{K}$. It satisfies the condition (0.1), and if 2 is not split in $K$, then $\mathfrak{A}$ is only ramified at the two real places corresponding to $\sigma_{2}$ and $\sigma_{3}$. Let $1, j, k, j k$ be a basis for $\mathfrak{A}$ like the one in (1.1). If $\mathcal{O}=R+R j+R k+R j k$ and $\mathcal{M}$ is a maximal order containing $\mathcal{O}$, then $[\mathcal{M}: \mathcal{O}]=4$.

Now we fix $\omega$ to be the positive root of the polynomial

$$
f(x)=x^{3}+2 x^{2}-x-1 .
$$

Then all the conditions above are satisfied. The field $K$ is the unique cubic Galois field with discriminant equal to 49 . We fix an embedding of $\mathfrak{A}$ into $M_{2}(\mathbb{R})$ by setting

$$
j=\left[\begin{array}{cc}
\sqrt{\omega} & 0 \\
0 & -\sqrt{\omega}
\end{array}\right] \quad \text { and } \quad k=\left[\begin{array}{cc}
0 & 2 \\
-\frac{1}{2} & 0
\end{array}\right] .
$$

Let $a=a_{1}+a_{2} \omega+a_{3} \omega^{2} \in R$ and similarly for $b, c, d$. It is not hard to verify that a maximal order $\mathcal{M}$ containing $\mathcal{O}$ is given by all elements satisfying

$$
\left\{\begin{array}{l}
t=\omega, s=-1, r_{1}=2, r_{2}=-\frac{1}{2}, r=2 \\
c_{1} \equiv a_{2}+a_{3}+b_{2}(\bmod 2), d_{1} \equiv a_{3}+b_{2}+b_{3}(\bmod 2), \\
c_{2} \equiv a_{1}+a_{2}+b_{2}+b_{3}(\bmod 2), d_{2} \equiv a_{1}+a_{3}+b_{1}+b_{2}(\bmod 2), \\
c_{3} \equiv a_{1}+a_{2}+a_{3}+b_{1}+b_{3}(\bmod 2), d_{3} \equiv a_{2}+b_{1}+b_{2}+b_{3}(\bmod 2),
\end{array}\right.
$$

where we again adopt the notation from the beginning of Section 4.

In the case of a cubic field $K$, we get two sets of inequalities like the one in (4.2). They correspond to the second and third embedding of $K$ into $\mathbb{R}$. Combining these with the inequalities (4.3), we get bounds on $a_{i}, b_{i}, c_{i}$ and $d_{i}$ for $i=1,2,3$. By using these, we get the fundamental domain in Figure 3.

The point $A$ is elliptic of order 7, $B$ is elliptic of order 3 , and the points $C$ and $C^{\prime}$ are identified and are elliptic of order 2 . The group $\overline{\mathcal{M}}^{1}$ is generated by the elements $g_{2}, g_{3}$ and $g_{7}$ satisfying the relations

$$
g_{2}^{2}=g_{3}^{3}=g_{7}^{7}=g_{2} g_{3} g_{7}=1,
$$

with coefficients given in Table 2.

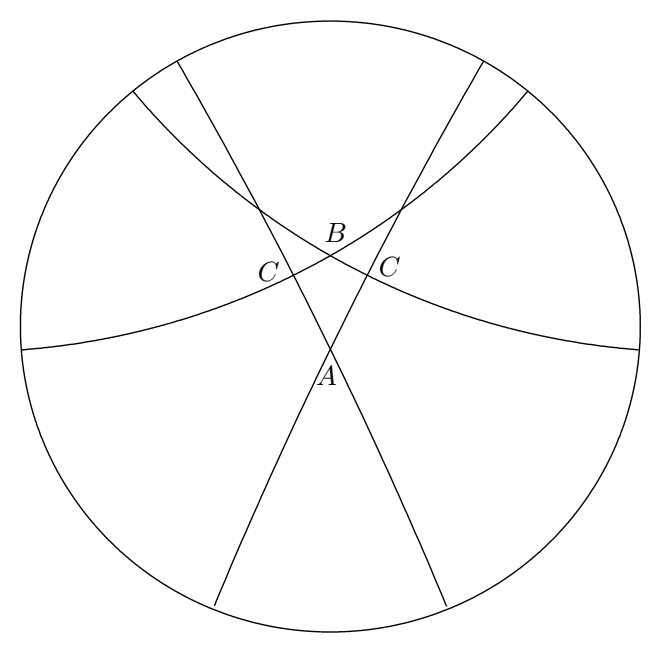

FIGURE 3. Fundamental domain in the case of a maximal order in $(\omega,-1)_{\mathbb{Q}(\omega)}$, where $\omega$ is the positive root of $x^{3}+2 x^{2}-x-1=0$. 
TABLE 2. Generators of the group corresponding to Figure 3.

\begin{tabular}{|c|c|c|c|c|c|c|c|c|c|c|c|c|}
\hline & $a_{1}$ & $a_{2}$ & $a_{3}$ & $b_{1}$ & $b_{2}$ & $b_{3}$ & $c_{1}$ & $c_{2}$ & $c_{3}$ & $d_{1}$ & $d_{2}$ & $d_{3}$ \\
\hline$q 2:$ & 0 & 0 & 0 & -2 & 1 & 1 & -1 & -2 & -1 & 2 & 1 & 0 \\
$q 3:$ & -1 & 0 & 0 & 0 & 0 & 0 & 0 & -3 & -1 & 2 & 1 & 0 \\
$q 7:$ & -1 & -1 & 0 & 0 & 0 & 0 & -1 & 0 & 0 & 2 & -1 & -1 \\
\hline
\end{tabular}

The element $g_{7}$ identifies the sides $A C$ and $A C^{\prime}$, and $g_{3}$ identifies the sides $B C$ and $B C^{\prime}$. We get a Riemann surface of genus 0 . Of course, the group $\overline{\mathcal{M}}^{1}$ is generated by $g_{3}$ and $g_{7}$ only, since $g_{2}=g_{3} g_{7}$.

The genus $g$ satisfies the following equality:

$$
2 g-2=\mu(\mathcal{F})-\sum_{q \geq 2} e_{q} \frac{q-1}{q},
$$

where $\mu(\mathcal{F})$ is the area of a fundamental domain and $e_{q}$ is the number of elliptic points of order $q$. If we combine this with (5.1) and our result, we get that $\zeta_{K}(-1)=-\frac{1}{21}$. This agrees with the result in [4].

\section{REFERENCES}

1. R. Aurich, E. B. Bogomolny, and F. Steiner, Periodic orbits on the regular hyperbolic octagon, Phys. D 48 (1991), 91-101. MR 92b:58173

2. Martin Eichler, Über die Idealklassenzahl hyperkomplexer Systeme, Math. Z. 43 (1938), 481494 .

3. Lester R. Ford, The fundamental region for a Fuchsian group, Bull. Amer. Math. Soc. 31 (1925), 531-539.

4. U. Halbritter and M. Pohst, On the computation of the values of zeta functions of totally real cubic fields, J. Number Theory 36 (1990), 266-288. MR 92b:11080

5. Svetlana Katok, Fuchsian groups, The University of Chicago Press, 1992. MR 93d:20088

6. Claiborne G. Latimer, On the fundamental number of a rational generalized quaternion algebra, Duke Math. J. 1 (1935), 433-435.

7. O.T. O'Meara, Introduction to quadratic forms, Springer-Verlag, Berlin-Heidelberg-New York, 1963. MR 27:2485

8. Marie-France Vigneras, Invariants numériques des groupes de Hilbert, Math. Ann. 224 (1976), 189-215. MR 55:2765

Department of Mathematics, Chalmers University of Technology and Göteborg UniVERSity, S-412 96 Göteborg, Sweden

Current address: Department of Mathematics, Institute for Advanced Study, Princeton, NJ 08540

E-mail address: sj@math.chalmers.se 